\title{
EXCHANGE DE RISQUES ENTRE ASSUREURS ET THEORIE DES JEUX
}

\author{
JEAN LEMAIRE \\ Bruxelles \\ RÉSUMÉ
}

Un théorème de Borch caractérisant les traités d'échange de risques Pareto-optimaux d'un marché de réassurance est étendu au cas d'utilités non différentiables. Les conditions d'existence d'une solution aux équations sont étudiées. Yous montrons ensuite que le marché constitue en fait un jeu coopératif a $m$ joueurs a utilités non-tranférables. Nous déterminons un contrat optimal de réassurance, d'abord en calculant la valeur au sens de Shapley du jeu, puis en introduisant un nouveau concept de valeur. Les deux techniques sont illustrées au moyen d'un exemple.

\section{SUMMARY}

A theorem of Borch characterizing Pareto-optimal treaties in a reinsurance market is extended to the case of non-differentiable utilities. Sufficient conditions for the existence of a solution to the equations are established. The problem is then shown to be identical to the determination of the value of a cooperative non-transferable $m$-person game. We show how to compute the Shapley value of this game, then we introduce a new value concept. An example illustrates both methods.

\section{\$I. INTRODUCTION}

Considérons un marché de $m$ compagnies d'assurances $C_{1}, \ldots$, $C_{m}$. Désignons par $S_{j}$ le montant dont dispose $C_{j}$ pour régler les sinistres et par $F_{j}\left(x_{j}\right)$ la fonction de répartition du montant total des sinistres pour l'ensemble du portefeuille de $C_{j}$ pour toute la période considérée. La situation de $C_{j}$ peut être caractérisée par le couple $\left[S_{f}, F_{f}\left(x_{f}\right)\right]$.

Nous supposons que chaque compagnie évalue sa situation au moyen d'une fonction d'utilité

$$
U_{f}\left(x_{j}\right)=U_{f}\left[S_{f}, F_{f}\left(x_{j}\right)\right]=\int_{0}^{\infty} u_{f}\left(S_{j}-x_{j}\right) d F_{f}\left(x_{j}\right),
$$

où $u_{j}\left(S_{j}-x_{j}\right)$ représente l'utilité attachée à un montant monétaire $S_{j}-x_{j}$.

Bien entendu, toute fonction d'utilité ne convient pas pour décrire le comportement d'un assureur. C'est pourquoi nous limitons la classe de ces fonctions en formulant les hypothèses suivantes: 
I) $u_{j}(x)$ est une fonction non-décroissante de $x$ (un gain élevé est toujours préféré à un gain plus faible);

2) $u_{j}(x)$ est une fonction concave (ou, ce qui revient au même, chaque membre du marché a une aversion au risque positive. In $_{n}$ assureur a en effet toujours peur du risque, c'est pour cela qu'il se réassure. Si une compagnie avait une aversion au risque négative, ne fùt-ce qu'en un seul point, le problème serait trivial car elle serait disposée à distribuer ses réserves pour reprendre les portefeuilles de ses partenaires);

3) $u_{j}(x)$ est une fonction bornée supérieurement dans un intervalle ouvert contenant

$$
I=\left[-\infty, \sum_{i=1}^{m} S_{j}\right]
$$

(aucun traité de réassurance ne peut apporter une satisfaction infinie).

Les différents membres du marché vont chercher à augmenter leur utilité en concluant un traité d'échange de risques:

$$
\bar{v}=\left[y_{1}\left(x_{1}, \ldots, x_{m}\right), \ldots, y_{m}\left(x_{1}, \ldots, x_{m}\right)\right],
$$

où $y_{j}\left(x_{1}, \ldots, x_{m}\right)$ est le montant que $C_{f}$ doit payer si les sinistres pour les différentes compagnies s'élèvent respectivement à $x_{1}, \ldots$, $x_{m}$. Comme tous les sinistres doivent être indemnisés, les $y_{j}\left(x_{1}, \ldots\right.$, $x_{m}$ ) doivent satisfaire à la condition d'admissibilité

$$
\begin{aligned}
\sum_{1=1}^{m} y_{j}\left(x_{1}, \ldots, x_{m}\right) & =\sum_{,-1}^{m} x_{j} \\
& =z
\end{aligned}
$$

le montant total des sinistres.

Après signature du traité, l'utilité de $C_{j}$ devient

$$
U_{j}(\bar{y})=\int_{0} u_{j}\left[S_{j}-y_{j}(\bar{x})\right] d F(\bar{x}),
$$

où $\vec{x}=\left(x_{1}, \ldots, x_{m}\right), F(\vec{x})$ est la fonction de répartition liée de $\bar{x}$ et $\theta$ l'orthant positif de l'espace euclidien à $m$ dimensions.

Un traité $\bar{y}^{\prime}$ est dit préférable à $\bar{y}$ si

$$
U_{j}\left(\bar{v}^{\prime}\right) \geq U_{j}(\bar{y}) \quad \forall j .
$$

avec le signe d'inégalité strict pour au moins un $j$. 
Un traité $\bar{y}$ est Pareto-optimal s'il n'en existe aucun qui lui soit préférable. En d'autres termes $\bar{y}$ est Pareto-optimal si et seulement si

$$
U_{j}\left(\bar{y}^{\prime}\right) \geq U_{j}(\bar{y}) \quad \forall j
$$

implique

$$
U_{j}\left(\bar{y}^{\prime}\right)=U_{j}\left(\bar{y}^{\prime}\right) \quad \forall j .
$$

Un traité Pareto-optimal représente un équilibre stable dans le marché. Un traité additionnel conclu dans cette situation ne pourrait élever l'utilité d'une compagnie sans faire décroître celle d'au moins un partenaire.

Borch ([I] à [4]), puis Du . Iouchel ([5]) ont démontré un théorème permettant de caractériser les traités Pareto-optimaux au moyen d'un ensemble de $m$ - I constantes. Cependant, ces auteurs utilisent certaines propriétés de dérivabilité des fonctions d'utilité qui ne possèdent aucune justification économique; c'est pourquoi nous allons généraliser le théorème au cas d'utilités non-différentiables.

\section{\$2. Caractérisation et Existence des Traites PARETO-OPTIMAUX}

THÉORÈne I: Un traité $\bar{y}$ est Pareto-optimal si et seulement si il existe $m$ constantes non-négatives $k_{1}, k_{2}, \ldots, k_{m}$ telles que, avec une probabilité $I$,

$$
k_{j} u_{j}^{\prime}\left[S_{j}-y_{j}(\bar{x})\right]=k_{1} u_{1}^{\prime}\left[S_{1}-y_{1}(\bar{x})\right], \quad j=\mathrm{I}, \ldots, m
$$

$u_{j}^{\prime}+(x)$ désignant la dérivée à droite de $u_{j}(x)$.

L'énoncé a un sens car il est bien connu que toute fonction concave, bornée dans un intervalle ouvert, admet une dérivée à droite (ainsi qu'une dérivée à gauche) finies en tout point. Ces dérivées sont monotones non croissantes.

Démonstration. Condition suffisante: Soit un traité $\bar{y}^{\prime}=\bar{y}+\bar{e}$, où $\vec{e}=\left[e_{1}(\bar{x}), \ldots, e_{m}(\bar{x})\right]$, où au moins une des $e_{j}(\bar{x})$ est non nulle et supposons la relation (2) vérifiée pour $\bar{y}$. Nous allons montrer que $\bar{y}$ est Pareto-optimal.

Le cas où certains $k_{j}$ sont nuls est trivial: la compagnie $C_{j}$ ne peut espérer améliorer son utilité et son cas ne doit pas être envisagé. 
Nous pouvons donc supposer les $k_{j}$ positifs. Choisissons un indice $j$ tel que $e_{j}(\bar{x})$ soit non nulle. Il vient

$U_{j}\left(\bar{y}^{\prime}\right)-U_{j}(y)=\int_{\theta}\left\{u_{j}\left[S_{j}-y_{j}(\bar{x})-e_{j}(\bar{x})\right]-u_{j}\left[S_{j}-y_{j}(\bar{x})\right]\right\} d F(\bar{x})$.

Nous devons distinguer le cas où $e_{j}(\bar{x})$ est positive de celui où cette quantité est négative.

Supposons d'abord $e_{j}(\vec{x})$ positive. Par définition de la dérivée $a$ gauche

$$
u_{j}^{\prime}(x)=\lim _{e_{i}(\bar{x}) \rightarrow 0} \frac{u_{j}\left[S_{j}-y_{j}(\bar{x})-e_{j}(\bar{x})\right]-u_{j}\left[S_{j}-y_{j}(\bar{x})\right]}{-e_{j}(\bar{x})},
$$

il vient

$$
U_{f}\left(\bar{y}^{\prime}\right)-U_{f}(\bar{y})=\int_{\theta}\left\{-e_{j}(\bar{x}) u_{j}^{\prime}\left[S_{j}-y_{j}(\bar{x})\right]+\theta_{j}\left[e_{j}(\bar{x}), y_{j}(\bar{x})\right]\right\} d F(\bar{x}),
$$

où

$$
\begin{array}{r}
\theta_{j}\left[e_{j}(\bar{x}), y_{j}(\bar{x})\right]=u_{j}\left[S_{j}-y_{j}(\bar{x})-e_{j}(\bar{x})\right]-u_{j}\left[S_{j}-y_{j}(\bar{x})\right]+ \\
+e_{j}(\bar{x}) u_{j}^{\prime}-\left[S_{j}-y_{j}(\bar{x})\right] .
\end{array}
$$

Nous allons montrer que $\theta_{j}\left[e_{j}(\vec{x}), u_{j}(\vec{x})\right]$ est une quantité nonpositive, c'est-à-dire que

$$
\frac{u_{j}\left[S_{j}-y_{j}(\bar{x})\right]-u_{j}\left[S_{j}-y_{j}(x)-e_{j}(\bar{x})\right]}{e_{j}(\bar{x})} \geqslant u_{j}^{\prime}-\left[S_{j}-y_{j}(\bar{x})\right] .
$$

ou, ce qui revient au même,

$$
\begin{aligned}
& \frac{u_{j}\left[S_{j}-y_{j}(\bar{x})\right]-u_{j}\left[S_{j}-y_{j}(\bar{x})-e_{j}(\bar{x})\right]}{e_{j}(\bar{x})} \geqslant \\
& \geqslant \frac{u_{j}\left[S_{j}-y_{j}(\bar{x})\right]-u_{j}\left[S_{j}-y_{j}(\bar{x})-\alpha e_{j}(\bar{x})\right]}{\alpha e_{j}(\bar{x})} \quad 0<\alpha \leqslant \mathrm{I}
\end{aligned}
$$

ou

$$
\begin{aligned}
u_{j}\left[S_{j}-y_{j}(\bar{x})\right]-u_{j}\left[S_{j}-y_{j}(x)-\alpha e_{j}(x)\right] \leqslant & \alpha\left\{u_{j}\left[S_{j}-y_{j}(\bar{x})\right]-\right. \\
& \left.-u_{j}\left[S_{j}-y_{j}(x)-e_{j}(\bar{x})\right]\right\} .
\end{aligned}
$$

Cette inégalité résulte de la concavité de la fonction d'utilité. 
Par conséquent

$$
U_{j}\left(\bar{y}^{\prime}\right)-U_{j}(\bar{y}) \leqslant-\int_{\theta} e_{j}(\bar{x}) u_{j}^{\prime}\left(\left[S_{j}-y_{j}(\bar{x})\right] d F(\bar{x}) .\right.
$$

La concavité de $u_{j}(x)$ implique également

$$
u u_{j}^{\prime}\left[S_{j}-y_{j}(\bar{x})\right] \geqslant u_{j}^{\prime}\left[S_{j}-y_{j}(\bar{x})\right] .
$$

Donc

$$
U_{j}\left(\bar{y}^{\prime}\right)-U_{j}(\bar{y}) \leqslant-\int_{0} e_{j}(\bar{x}) u_{j}^{\prime}{ }^{+}\left[S_{j}-y_{j}(\bar{x})\right] d F(\bar{x}) .
$$

En utilisant (2)

$$
U_{j}\left(\bar{y}^{\prime}\right)-U_{j}(\bar{y}) \leqslant-\frac{k_{1}}{k_{j}} \int_{\theta} e_{j}(\bar{x}) u_{1}^{\prime}+\left[S_{1}-y_{1}(\bar{x})\right] d F(\bar{x}) .
$$

La même inégalité peut se démontrer dans le cas $e_{j}(\bar{x})<0$, en employant directement des dérivées à droite.

Puisque $\sum_{i=1}^{m} y_{j}(\bar{x})=\sum_{i=1}^{m} y_{j}^{\prime}(\bar{x})=\sum_{i=1}^{m} x_{j}$, il faut que $\sum_{i=1}^{m} e_{j}(x)=0$.

Il vient, en multipliant par $k_{j}$ et en sommant $\sum_{i=1}^{m} k_{j}\left[U_{j}\left(\bar{y}^{\prime}\right)-U_{j}(\bar{y})\right] \leqslant-k_{1} \int_{0} u_{1}^{\prime}+\left[S_{1}-y_{1}(\bar{x})\right] \sum_{i=2}^{m} e_{j}(\bar{x}) d F(\bar{x})=0$.

Si $\bar{y}$ n'est pas Pareto-optimal, il doit exister un $\bar{y}^{\prime}$ tel que chaque terme du membre de gauche soit non-négatif, avec au moins un terme positif, ce qui est impossible. Donc $\bar{y}$ est Pareto-optimal.

Condition nécessaire: Supposons par exemple qu'il n'existe pas de $k_{1}$ et $k_{2}$ telles que la relation (2) soit vérifiée pour un traité $\bar{y}$. Nous allons construire un traité $\bar{y}^{\prime}$ meilleur que $\bar{v}$.

$$
\text { Posons s'abord } k=\frac{\int_{0} u_{1}^{\prime}+\left[S_{1}-y_{1}(\bar{x})\right] u_{2}^{\prime}+\left[S_{2}-y_{2}(\bar{x})\right] d F(\bar{x})}{\int_{0}^{\{}\left\{u_{1}^{\prime}+\left[S_{1}-y_{1}(\bar{x})\right]\right\}^{2} d F(\bar{x})}
$$

et

$$
\nu(\bar{x})=u_{2}^{\prime}+\left[S_{2}-y_{2}(x)\right]-k u_{2}^{\prime}+\left[S_{1}-y_{1}(\bar{x})\right] .
$$


Alors

$$
\begin{aligned}
\int_{\theta} v(\bar{x}) u_{1}^{\prime}+\left[S_{1}-y_{1}(\bar{x})\right] d F(\bar{x})= & \int_{\theta}\left\{u_{2}^{\prime}+\left[S_{2}-y_{2}(\bar{x})\right]-k u_{1}^{\prime}+\left[S_{1}-y_{1}(\bar{x})\right] \cdot\right. \\
& u_{1}^{\prime}\left[S_{1}-y_{1}(\bar{x})\right] d F(\bar{x}) \\
= & \int_{\theta} u_{1}^{\prime}+\left[S_{2}-y_{2}(\bar{x})\right] u u_{1}^{\prime}\left[S_{1}-y_{1}(\bar{x})\right] d F(\bar{x}) \\
& \quad-k \int_{0}\left\{u_{1}^{\prime}\left[S_{1}-y_{1}(\bar{x})\right]\right\}^{2} d F(\bar{x}) \\
= & 0 .
\end{aligned}
$$

$v(\bar{x})$ est donc la partie de $u_{2}^{\prime}+\left[S_{2}-y_{2}(\bar{x})\right]$ orthogonale à $u_{1}^{\prime}{ }^{+}\left[S_{1}-y_{1}(\bar{x})\right]$.

Comme (2) n'est pas vérifiée pour $j=2$ et pour toutes $k_{1}, k_{2}$,

$$
\int_{\theta} v^{2}(\bar{x}) d F(\bar{x})>0 \text {. }
$$

Posons

$$
\delta=\frac{1}{2} \frac{\int_{\theta} \nu^{2}(\bar{x}) d F(x)}{\int_{0} u_{2}^{\prime}}+\frac{\theta}{\left[S_{2}-y_{2}(\bar{x})\right] d F(\bar{x})}>0 .
$$

Définissons le nouveau traité $\bar{y}^{\prime}=\bar{y}+\bar{e}$, où

$$
\begin{array}{llrl}
e_{1}(\bar{x}) & =[\eta(\bar{x})-\delta] \varepsilon ; & & (\varepsilon>0) \\
e_{2}(\bar{x}) & =-e_{1}(\bar{x}) ; & & \\
e_{j}(\bar{x}) & =0 & & (j>2) .
\end{array}
$$

En employant une technique similaire à (3),

$$
\begin{aligned}
& U_{1}^{\prime}\left(\bar{y}^{\prime}\right)-U_{1}(\bar{y})= \int_{\theta}\left\{-e_{1}(\bar{x}) u^{\prime}+\left[S_{1}-y_{1}(\bar{x})\right]+\theta_{1}\left[e_{1}(\bar{x}), y_{1}(\bar{x})\right]\right\} d F(\bar{x}) \\
&= \varepsilon\left\{\int_{\theta}-[v(\bar{x})-\delta] u_{1}^{\prime}+\left[S_{1}-y_{1}(\bar{x})\right] d F(\bar{x})+(\mathrm{I} / s)\right. \\
&\left.\int_{\theta} \theta_{1}^{+}\left[e_{1}(\bar{x}), y_{1}(\bar{x})\right] d F(\bar{x})\right\} \\
&= \varepsilon\left\{\delta \int_{\theta} u_{1}^{\prime}+\left[S_{1}-y_{1}(\bar{x})\right] d F(\bar{x})+(\mathbf{I} / \varepsilon)\right. \\
&\left.\int_{\theta} \theta_{1}^{+}\left[e_{1}(\bar{x}), y_{1}(\bar{x})\right] d F(\bar{x})\right\}
\end{aligned}
$$


en utilisant (4). Etudions le comportement du dernier terme du second membre au voisinage de $\varepsilon=\dot{0}$.

$$
\begin{aligned}
& (\mathrm{I} / \varepsilon) \theta_{1}^{+}\left[e_{1}(\bar{x}), y_{1}(\bar{x})\right]=(\mathrm{I} / \varepsilon)\left\{u_{1}\left[S_{1}-y_{1}(\bar{x})-e_{1}(\bar{x})\right]-u_{1}\left[S_{1}-y_{1}(\bar{x})\right]+\right. \\
& +e_{1}(\bar{x}) u_{1}^{\prime}+\left[S_{1}-y_{1}(\bar{x})\right] \\
& =(I / \varepsilon)\left\{u_{1}\left[S_{1}-y_{1}(\bar{x})-(v(\bar{x})-\delta) \varepsilon\right]-u_{1}\left[S_{1}-y_{1}(\bar{x})\right]\right. \\
& \left.+(v(x)-\delta) \varepsilon u_{1}^{\prime}{ }^{+}\left[S_{1}-y_{1}(\bar{x})\right]\right\} \\
& =-[v(\bar{x})-\delta]\left\{\frac{u_{1}\left[S_{1}-\dot{y}(x)-(v(x)-\delta) \varepsilon\right]-u_{1}\left[S_{1}-y_{1}(\bar{x})\right]}{-(v(\bar{x})-\delta) \varepsilon}\right. \\
& \left.-u_{1}^{\prime}+\left[S_{1}-y_{1}(\bar{x})\right]\right\} \text {. }
\end{aligned}
$$

Il nous faut à nouveau distinguer deux cas suivant le signe de $[v(\tilde{x})-\delta] \varepsilon$.

Si cette quantité est positive, le terme

$$
\frac{u_{1}\left[S_{1}-y_{1}(x)-(v(x)-\delta) \varepsilon\right]-u_{1}\left[S_{1}-y_{1}(x)\right]}{-(v(\bar{x})-\delta) \varepsilon}
$$

tend vers $u_{1}^{\prime}-\left[S_{1}-y_{1}(\vec{x})\right]$ lorsque $\varepsilon \rightarrow 0$. Posons

$$
u_{1}^{\prime}-\left[S_{1}-y_{1}(x)\right]-u_{1}^{\prime}+\left[S_{1}-y_{1}(x)\right]=\beta_{1}>0 .
$$

L'expression entre accolades tend vers $\beta_{1}$. Cette convergence est monotone puisque les dérivées sont des fonctions monotones de $y_{1}(\bar{x})$. Donc

$$
\begin{aligned}
& \lim _{\varepsilon \rightarrow 0}(\mathrm{I} / \varepsilon) \int_{\theta} \theta_{1}^{+}\left[e_{1}(\bar{x}), y_{1}(\bar{x})\right] d F(\bar{x})=\int \lim _{\theta \rightarrow 0}(\mathrm{I} / \varepsilon) \theta_{1}^{+}\left[e\left(\bar{x}_{1}\right), y_{1}(\bar{x})\right] d F(\bar{x}) \\
& =\int_{\theta} \beta_{1} d F(\bar{x}) \\
& =\beta_{1} \text {. } \\
& U_{1}\left(\bar{y}^{\prime}\right)-U(\bar{y})=\varepsilon\left\{\delta \int_{\theta} u_{1}^{\prime}+\left[S_{1}-y_{1}(\bar{x})\right] d F(\bar{x})+\beta_{1}\right\} .
\end{aligned}
$$

Comme $\delta>0$ et $\int_{\theta} u_{1}^{\prime}+\left[S_{1}-y_{1}(x)\right] d F(x)>0, U_{1}\left(\bar{y}^{\prime}\right)-U_{1}(\bar{y})$ est positive pour $\varepsilon$ suffisamment petit.

Lorsque $[v(\bar{x})-\delta] \varepsilon$ est négative, l'expression entre accolades tend vers zéro, ce qui ne change rien à la conclusion. 
De manière similaire nous avons pour $C_{2}$ :

$$
\begin{aligned}
& U_{2}\left(\bar{y}^{\prime}\right)-U_{2}(\bar{y})=\int_{\theta}\left\{-e_{2}(\bar{x}) u_{2}^{\prime}+\left[S_{2}-y_{3}(\bar{x})\right]+\theta_{2}^{+}\left[e_{2}(x), y_{2}(x)\right]\right\} d F(\bar{x}) \\
& =\varepsilon \int_{0}[y(\bar{x})-\delta] i_{2}^{\prime}+\left[S_{2}-y_{2}(\bar{x})\right] d F(\bar{x})+ \\
& \left.+(I / \Sigma) \int_{0} \theta_{2}^{+}\left[e_{3}(\bar{x}), y_{2}(\bar{x})\right] d F(\bar{x})\right\} \\
& =\varepsilon\left\{\int_{\theta} \nu(\bar{x})\left\{k u_{1}^{\prime}+\left[S_{1}-y_{1}(\bar{x})\right]+\nu(\bar{x})\right\} d F(\bar{x})-\right. \\
& -\delta \int_{\theta} u_{2}^{\prime+}\left[S_{2}-y_{2}(\bar{x})\right] d F(\bar{x}) \\
& \left.+(\mathrm{I} / \mathrm{s}) \int_{0} \theta_{2}^{-}\left[e_{2}(\bar{x}), y_{2}(\bar{x})\right] d F(x)\right\} \\
& =\varepsilon\left\{\int_{0} \nu^{2}(\bar{x}) d F(\bar{x})-\delta \int_{0} u_{2}^{\prime}+\left[S_{2}-y_{2}(\bar{x})\right] d F(\bar{x})\right. \\
& \left.+(\mathrm{r} / \varepsilon) \int_{0} \theta_{2}^{+}\left[e_{2}(\bar{x}), y_{2}(\bar{x})\right] d F(x)\right\} \\
& =\varepsilon\left\{\frac{1}{2} \int \nu^{2}(\bar{x}) d F(\bar{x})+(I / \varepsilon) \int \theta_{2}^{+}\left[e_{2}(\bar{x}), y_{2}(\bar{x})\right] d F(\bar{x})\right\}
\end{aligned}
$$

en utilisant (4).

La dernière intégrale peut à nouveau être remplacée, soit par zéro, soit par

$$
\hat{\beta}_{2}=u \iota_{2}^{\prime}-\left[S_{2}-y_{3}(\bar{x})\right]-u_{2}^{\prime}+\left[S_{2}-y_{2}(\bar{x})\right]>0 .
$$

Il en résulte que $U_{2}\left(\bar{y}^{\prime}\right)-U_{2}(\bar{y})>0$ pour $\varepsilon$ suffisamment petit.

Puisque

$$
\begin{array}{ll}
U_{j}\left(\bar{y}^{\prime}\right)>U_{j}(\bar{y}) & \text { pour } j=\mathrm{r}, 2 \\
U_{j}\left(\bar{y}^{\prime}\right)=U_{j}(\bar{y}) & \text { pour tout } j>2
\end{array}
$$

$\bar{y}$ n'est pas Pareto-optimal.

Remarquons que la condition suffisante est vraie que nous prenions des dérivées à gauche ou à droite. L'obligation d'employer des dérivées à droite dans la condition nécessaire résulte de la concavité des $u \iota_{j}(x)$.

$\mathrm{Si}$ le théorème précédent permet de caractériser les traités Pareto-optimaux, il n'assure pas l'existence d'une solution aux équations (2). Les théorèmes et le contre-exemple suivants apportent une réponse à cette question. 
ThÉORÈME 2. (Du Mouchel) Si les fonctions d'utilité sont dérivables en tout point d'un intervalle contenant $I=\left(\infty, \sum_{j=1}^{m} S_{f}\right]$ et si les $k_{f}$ peuvent être choisis tels que les domaines des fonctions $u_{j}^{\prime}(x) . k_{f}$ ont une intersection non-vide, il existe une solution Paretooptimale.

ThÉorène 3. Pour un ensemble de constantes $k_{j}$ fixé, satisfaisant aux hypothèses du théorème 2 , il existe un et un seul traité Pareto-optimal.

Démonstration: L'existence d'un traité étant assurée par le théorème 2, il suffit de démontrer l'unicité.

Les relations (2) définissent implicitement les $y_{j}(\bar{x})$ en fonction de $y_{1}(\bar{x})$, pour $z$ fixé. Soient

$$
y_{j}(\bar{x})=\gamma_{j}\left(y_{1}(\bar{x})\right) \quad j=2, \ldots, m
$$

ces fonctions. Comme les $u_{j}^{\prime}(x)$ sont des fonctions continues nondécroissantes des $y_{j}(\bar{x})$, les $\gamma_{j}\left(y_{3}(\bar{x})\right)$ sont des fonctions uniformes, continues et non-décroissantes. La condition d'admissibilité

devient

$$
\sum_{i=1}^{m} y_{j}(\bar{x})=\sum_{i=1}^{m} x_{j}=z
$$

$$
y_{1}(\bar{x})+\sum_{i=:}^{m} \gamma_{j}\left(y_{1}(\bar{x})\right)=z .
$$

Le premier membre est une fonction continue croissante de $y_{1}(\bar{x})$. Donc pour tout $z$ il existe un et un seul traité $y_{1}(\bar{x})$ Paretooptimal. Un mème argument peut être répété pour les autres compagnies.

ThÉORÈm 4. Pour un ensemble de constantes $k_{j}$ fixé, il existe au plus un traité Pareto-optimal.

La démonstration s'appuie sur un raisonnement analogue à celui du théorème 3 , utilisant des dérivées à droite. La non-continuité des $u_{f}^{\prime}(\bar{x})$ en les points où les fonctions d'utilité ne sont pas dérivables implique qu'il peut ne pas $y$ avoir de solution admissible aux équations (2), comme le confirme le contre-exemple suivant. 
Soient

$$
\begin{aligned}
& \imath_{1}(x)=\mathrm{I}-e^{\left(x-S_{1}\right)} \\
& \iota_{2}(x)=\left\{\begin{array}{ll}
a\left(x-S_{2}\right) & x<S_{2} \\
b\left(x-S_{2}\right) & x \geqslant S_{2} .
\end{array} \quad a>b>0\right.
\end{aligned}
$$

Alors

$$
\begin{aligned}
& u_{1}^{\prime}\left[S_{1}-y_{1}(\bar{x})\right]=e^{y_{2}(\bar{x})} \\
& u_{2}^{\prime}+\left[S_{2}-y_{2}(\bar{x})\right]= \begin{cases}a & y_{2}(\bar{x})>0 \\
b & y_{2}(\bar{x}) \leqslant 0 .\end{cases}
\end{aligned}
$$

La relation (2) devient

$$
\begin{aligned}
& \int k_{2} a=k_{1} e^{y_{1}(\bar{x})} \quad y_{2}(\bar{x})>0 \\
& k_{2} b=k_{1} e^{y_{1}(\bar{x})} \quad y_{2}(\bar{x}) \leqslant 0 \\
& \int \log k_{2}+\log a=\log k_{1}+y_{1}(\bar{x}) \quad y_{2}(\bar{x})>0 \\
& \left(\log k_{2}+\log b=\log k_{1}+y_{1}(\bar{x}) \quad y_{2}(\bar{x}) \leqslant 0\right. \\
& \int y_{1}(\bar{x})=\log a+\log k_{2}-\log k_{1}=A \quad y_{2}(\bar{x})>0 \\
& \left\langle y_{1}(\bar{x})=\log b+\log k_{2}-\log k_{1}=B \quad y_{2}(\bar{x}) \leqslant 0\right.
\end{aligned}
$$

avec $A>B$.

Or $y_{2}(\bar{x})=z-y_{1}(\bar{x})$. Donc $y_{2}(\bar{x})>0$ implique $y_{1}(\bar{x})<z$ et $y_{2}(\bar{x}) \leqslant 0 \rightarrow y_{1}(\bar{x}) \geqslant z$. Donc

$$
\begin{cases}y_{1}(\bar{x})=A & y_{1}(\bar{x})<z \\ y_{1}(\bar{x})=B & y_{1}(\bar{x}) \geqslant z .\end{cases}
$$

Alors $z>y_{1}(\bar{x})=A>B=y_{1}(\bar{x}) \geqslant z$ ce qui est une contradiction.

THÉORÈME 5. Si les fonctions d'utilité sont strictement concaves et si les $y_{j}(\bar{x})$ sont différentiables, un traité Pareto-optimal ne dépend des montants des sinistres $x_{j}$ que par l'intermédiaire de leur somme $z=\sum_{i=1}^{m} x_{j}$. 
Cette propriété constitue une généralisation d'un théorème de Borch ([3]). La démonstration en est semblable, à condition d'utiliser les dérivées secondes à droite $\left(u_{j}^{\prime}\right)^{\prime}+(x)$, qui existent car les $u u_{j}^{\prime}(x)$ sont des fonctions monotones. Le théorème signifie que le montant payé par $C_{j}$ ne dépend que de la somme des sinistres à régler sur l'ensemble du marché. Sous les conditions de l'énoncé, tout traité Pareto-optimal revient à former un pool de toutes les compagnies et décider d'une règle pour la répartition des charges: les assureurs ont donc toujours intérêt à coopérer.

\section{§3. LeMarche de Réassurance en tant que Jeu a Utilites NON-TRANSFÉRABLES}

Nous allons dorénavant supposer qu'il existe un traité Paretooptimal, fourni par les équations (I) et (2). Ce traité est unique lorsque les constantes $k_{j}$ sont déterminées (théorème 3 ). Cependant, il existera en général tout un domaine de $k_{j}$ fournissant une solution admissible. Les $k_{j}$ ne sont déterminées qu'à un facteur près: (2) n'est pas modifiée lorsque les $k_{j}$ sont multipliées par une constante. Nous pouvons donc arbitrairement restreindre le domaine des $k_{j}$, par exemple en posant $k_{1}=\mathrm{I}$ ou $\sum_{i=1}^{m} k_{j}=m$.

Dans l'espace euclidien à $m$ dimensions formé par les utilités des compagnies, l'ensemble des $k_{f}$ admissibles forme une surface à $m-$ I dimensions, appelée surface Pareto-optimale. Ses équations paramétriques (en les paramètres $k_{1}, \ldots, k_{m}$ ) sont

$$
U_{j}=\int_{\theta} u_{j}\left[S_{f}-y_{j}(\bar{x})\right] d F(\bar{x}), \quad j=I, \ldots, m
$$

où les $y_{f}(\bar{x})$ satisfont aux relations (2).

Une compagnie n'acceptera de faire partie du pool que si cela entraîne pour elle une amélioration de sa situation, c'est-à-dire une augmentation de son utilité. La surface Pareto-optimale est donc limitée par les $m$ hyperplans d'équations

$$
U_{j}=U_{j}\left(x_{j}\right) .
$$

L'espace délimité par la surface Pareto-optimale et les $m$ hyperplans est appelé l'espace du jeu $\xi$. 
Toutes ces considérations suggèrent en effet une analogie avec la théorie coopérative des jeux. Nous allons montrer que le problème tel que nous l'avons formulé constitue en fait un jeu à $m$ joueurs à utilités non-transférables.

Définition: Un jeu à utilités non-transférables est défini par un triplet $[M, v(S), H]$, où

I) $M$ est un ensemble fini d'éléments (les joueurs);

2) $v(S)$ est une fonction (appelée fonction caractéristique) définie sur tous les sous-ensembles non-vides $S$ de $M$, envoyant chaque $S$ (les coalitions) sur un sous-ensemble $v(S)$ de l'espace euclidien à $|S|$ dimensions, tel que

a) $v(S)$ est non-vide;

b) $v(S)$ est convexe;

c) $v(S)$ est fermée;

d) $v(S)$ est suradditive: $\forall S_{1}, S_{2} \subset M$, $\supset-S_{1} \cap S_{2}=\phi, \quad v\left(S_{1} \cup S_{2}\right) \supset v\left(S_{1}\right) \times v\left(S_{2}\right)$;

3) $H$ est ,l'ensemble des résultats réellement accessibles". Plus précisément:

$$
\tau^{\prime}(M)=\left\{r \varepsilon E^{|M|} \mid \exists y \varepsilon H \supset-y \geqslant x\right\} .
$$

Soient I) $M$ l'ensemble de $m$ compagnies;

2) $v(S)$ l'espace délimité par la projection de la surface Pareto-optimale dans l'espace euclidien à $|S|$ dimensions;

3) $H=\xi$.

THÉORÈME 5. Le marché de réassurance est un jeu à utilités nontransférables $[M, v(S), \zeta]$.

Démonstration: Il suffit de montrer que $v(S)$ vérifie les propriétés a) à d).

a) $v(S)$ est non-vide: elle comporte certainement le point initial

$$
y_{j}(\bar{x})=x_{j} . \quad \forall j
$$

b) $v(S)$ est convexe: soient $\bar{y}^{\prime} S$ et $\bar{y}^{\prime \prime} S$ deux traités admissibles pour une coalition $S$. Nous avons donc

$$
\Sigma y_{j}^{\prime} S(x)=\Sigma y_{j}^{\prime \prime} S(x)=\Sigma x_{j}
$$


Le traité $\bar{y}^{S}=\alpha \bar{y}^{\prime} S+(\mathrm{I}-\alpha) \bar{y}^{\prime \prime}, S$ est également admissible car

$$
\begin{aligned}
& U_{j}(\bar{y})=U_{j}\left[\alpha \bar{y}^{\prime} S+(\mathrm{I}-\alpha) \bar{y}^{\prime \prime} S\right] \\
& =\alpha U_{j}\left(\bar{y}^{\prime} S\right)+(I-\alpha) U_{j}\left(\bar{y}^{\prime \prime} S\right) \text { en vertu de la pro- } \\
& \geqslant \alpha U_{j}\left(x_{j}\right)+(I-\alpha) U_{j}\left(x_{j}\right) \\
& =U_{j}\left(x_{j}\right) \text {. }
\end{aligned}
$$$$
\text { priété de linéarité des }
$$$$
\text { fonctions d'utilité }
$$

Le jeu est dit ,à utilités non-transférables" par opposition aux jeux à utilités transférables, pour lesquels les paiements latéraux entre joueurs sont autorisés et n'ont pas d'effet sur la somme des utilités de tous les joueurs. Ceci implique que les $u_{f}(x)$ sont de la forme $a_{j} x+b_{j}$, et que la surface Pareto-optimale est un plan d'éçuation

$$
\sum_{i=1}^{m} U_{j}=\text { constante. }
$$

Le jeu est dit inessentiel lorsque $\zeta$ se réduit à un point, à savoir le traité $y_{j}(\bar{x})=x_{j}$ pour tout $j$. De tels jeux sont intéressants car les joueurs ne peuvent retirer aucun bénéfice de leur coopération, ils correspondent à des cas de dégénérescence: les variances de certains portefeuilles sont nulles par exemple. Pour éviter d'inutiles précautions de langage, nous supposerons dorénavant le jeu essentiel.

La figure suivante représente un espace de jeu possible pour un marché de deux compagnies.

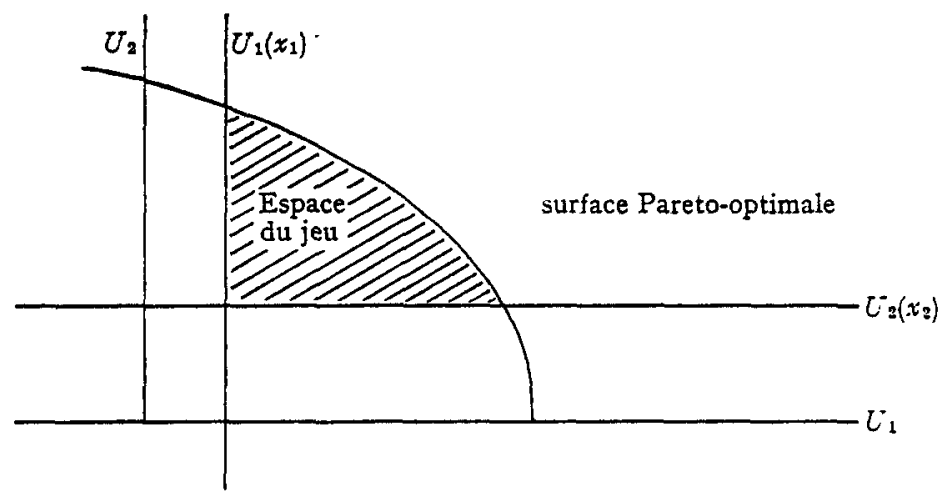


La solution Pareto-optimale n'est pas unique car nous avons choisi une définition d'optimalité assez faible. Il lui manque en effet certains axiomes de partage, précisant comment les joueurs vont répartir le bénéfice de leur coopération. Chaque compagnie a intérêt à obtenir une constante $k_{j}$ aussi grande que possible (compatible avec les conditions d'admissibilité) de manière à payer le moins possible. Le choix des $k_{j}$ dépend donc d'un marchandage supplémentaire, pendant lequel les intérêts des joueurs seront contradictoires. En termes de théorie des jeux, nous devons déterminer la valeur du jeu.

\section{\$4. VALEUR AU SENS DE SHAPLEY}

Le premier concept de valeur satisfaisant fut présenté par Shapley ([9]) en I953 dans le cadre des jeux à utilités transférables.

Définition: Le jeu à utilités transférables associé au marché $[M$, $v(S), \xi]$ est défini par le couple $[M, v(S)]$, où

I) $M$ est l'ensemble des joueurs;

2) $v(S)$ est une fonction d'ensemble, appelée fonction caractéristique, associant à toute coalition $S \subset . M$ l'hyperplan d'équation

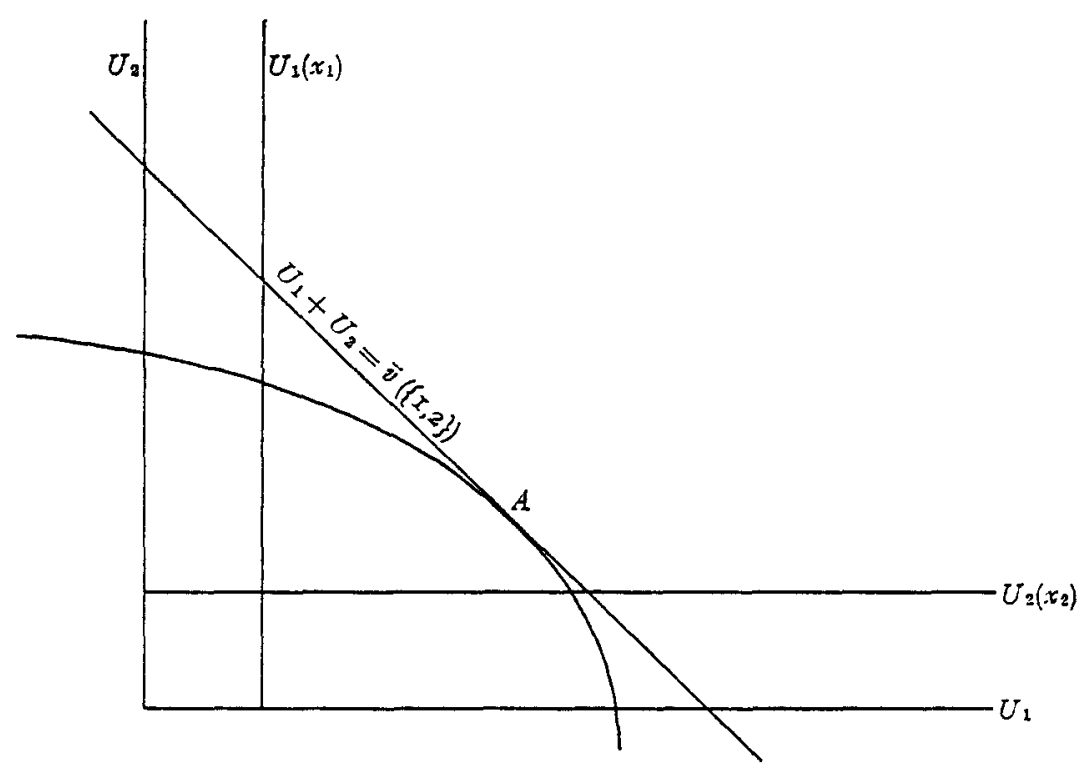


où

$$
\sum_{j \in s} U_{j}=\beta(S),
$$

$$
\beta(S)=\underset{U_{s^{2 \xi}}}{\max } \sum_{\text {ies }} U_{j} ; U_{S}=\left\{U_{j} \mid j \approx S\right\} .
$$

La figure de la page précédente représente le jeu à utilités transférables associé à une marché de deux compagnies.

Géométriquement, $\bar{v}(S)$ est l'hyperplan tangent à $v(S)$ dont tous les cosinus directeurs valent $\mathrm{I}$. Le point de tangence $A$ ne fait pas nécessairement partie de l'espace du jeu $\xi$; il peut conférer à un des joueurs une utilité inférieure à sa valeur initiale.

Remarquons que $A$ n'est pas nécessairement unique: l'intersection de $v(S)$ et $\bar{v}(S)$ pourrait être un segment de droite ou un morceau convexe d'hyperplain.

Une imputation - c'est-à-dire un partage du gain global - est un point $\bar{\phi}=\left(\phi_{1}, \ldots, \phi_{m}\right)$ tel que

$$
\begin{aligned}
& \phi_{j} \geqslant U_{j}\left(x_{j}\right) \\
& \sum_{i=1}^{m} \phi_{j}=\beta(M) .
\end{aligned}
$$$$
\forall j
$$

Shapley est parvenu à définir un concept de.valeur en isolant une imputation à partir de trois axiomes.

Soit $G_{m}$ l'ensemble de toutes les fonctions $\bar{v}(S)$.

Définition: On appelle fonction de valeur $\phi$, la fonction définie sur $G_{m}$ qui associe à toute $v(S) \varepsilon G_{m}$, une imputation

$$
\phi(\bar{v})=\left[\phi_{1}(\bar{v}), \ldots, \phi_{m}(\bar{v})\right],
$$

satisfaisant aux trois conditions suivantes:

I) Deux joueurs symétriques reçoivent le même montant;

Pour toute permutation $\pi$ de l'ensemble des jouleurs, et pour toute $\bar{v}(S)$ telle que $\bar{v}[\pi(S)]=\bar{v}(S)$ pour toute $S \subseteq M$,

$$
\phi_{\pi(j)}(v)=\phi_{j}(\bar{v}) . \quad j=I, \ldots, m .
$$

2) Un joueur inessentiel pour toute coalition ne bénéficie pas de la coopération:

S'il existe un $j \leq M$ tel que $\bar{v}(S)=\bar{v}(S-\{j\})+\bar{v}(\{j\})$ pour tout $S \subseteq M$ incluant $j$,

$$
\phi_{j}(\bar{v})=\bar{v}(\{j\}) .
$$


3) La fonction de valeur est linéaire.

$$
\begin{aligned}
& \text { Pour toutes } \bar{v}(S), \bar{w}(S)=G_{m}, \text { pour tous } a, b \\
& \phi_{j}(a \bar{v}+b \bar{w})=a \phi_{j}(\bar{v})+b \phi_{j}(\bar{w}) . \quad \jmath=I, \ldots, m .
\end{aligned}
$$

THÉORĖME 6. Il existe une et une seule fonction de valeur

$$
\begin{array}{r}
\phi_{j}(\bar{v})=\sum_{S \subseteq M}^{\sum} \frac{(s-I) !(m-s) !}{m !}[v(S)-\bar{v}(S-\{J\})] . \\
s=|S|
\end{array}
$$

La valeur au sens de Shapley peut être interprétée de la manière suivante: les joueurs entrent un par un dans la coalition, dans un ordre aléatoire. Chacun reçoit la totalité de ce qu'il apporte à la sous-coalition formée avant lui. Tous les ordres d'entrée sont envisagés, et résumé par une moyenne arithmétique.

Le modèle attribue donc à chacun l'espérance mathématique de sa valeur d'admission, lorsque toutes les permutations de joueurs sont équiprobables. Dans le cas d'un jeu à deux joueurs, la valeur au sens de Shapley $\phi(\vec{v})$ est le milieu du segment de droite $\bar{v}(\{I, 2\})$ limité par les utilités initiales: elle accorde le même gain d'utilité aux deux joueurs.

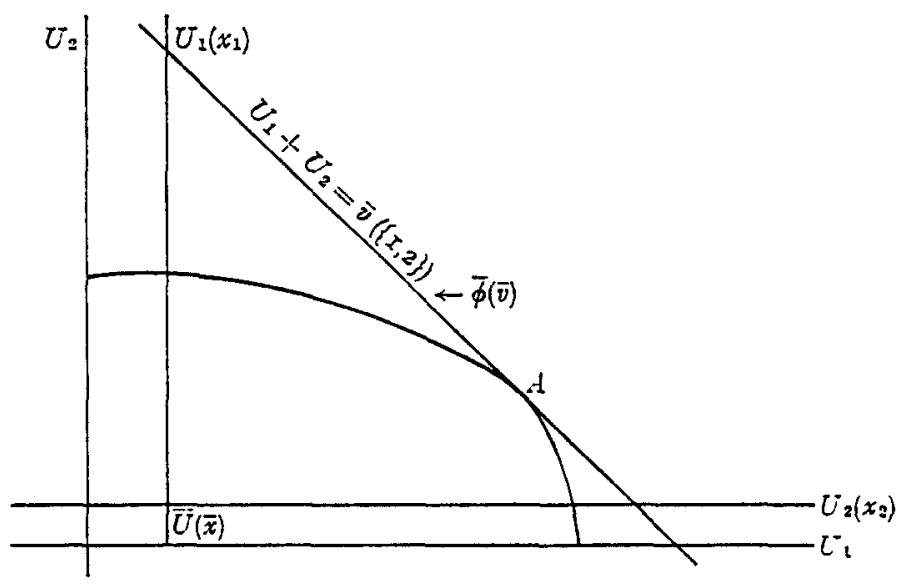

Alors se pose le problème important de généraliser ce concept de solution aux jeux à utilités non-transférables.

Le point $\phi(\bar{v})$ peut-il constituer une solution acceptable pour le marché de réassurance? Evidemment non car il se trouve en général 
en dehors de l'espace du jeu $\xi$ et ne peut donc être atteint par un traité. Ce n'est que dans le cas particulier où $\phi(\bar{v})$ coinciderait avec le point (ou un des points) de tangence $A$ qu'il pourrait être une solution valable (à condition que $A$ fasse partie de $\zeta$ ).

Or, les fonctions d'utilité des joueurs ne sont définies qu'à une transformation linéaire près. Nous pouvons donc multiplier ces fonctions par des constantes arbitraires non-négatives $k_{j}$. Cette opération a pour effet de modifier $\xi, \vec{v}(S)$, et par conséquent $A$ et $\phi(\vec{v})$.

Shapley ([Io]) a montré qu'il existe (au moins) une ensemble de "poids" $k_{f}$ tel que la valeur transformée fasse partie du nouvel espace du jeu. La démonstration peut être aisément adaptée au modèle de réassurance, et fournit dans ce cas un résultat complémentaire intéressant: les poids $k_{j}$ ne sont rien d'autre que les constantes apparaissant dans l'expression fondamentale (2). Ce résultat nous permet de donner une interprétation économique aux $k_{j}$.

Celles-ci représentent les forces relatives des joueurs.

$u_{j}^{\prime}\left[S_{j}-y_{j}(\bar{x})\right]$ est la pente de l'utilité de $C_{j}$ après règlement des sinistres. (2), qui peut s'écrire

$$
\frac{u_{j}^{\prime}+\left[S_{j}-y_{j}(\bar{x})\right]}{u_{i}^{\prime}+\left[S_{i}-y_{i}(\bar{x})\right]}=\frac{k_{i}}{k_{j}}, \quad \forall i, j
$$

exprime que ces pentes sont commensurables au moyen de "taux de change d'équilibre" $k_{i} / k_{j}$. Infinitésimalement, le marché peut être considéré comme un jeu à utilités transférables où les compagnies utilisent des ",monnaies" différentes. La solution ne change pas si localement $C_{\imath}$ et $C_{j}$ s'échangent de l'argent au taux $k_{i} / k_{j}, C_{j}$ devant donner $k_{i}$ unités pour en recevoir $k_{j}$. L'analogie avec les équilibres monétaires peut encore être poussée plus loin: si $C_{j}$ veut échanger un montant important avec $C_{i}$, il exerce une ,demande" sur les réserves de son partenaire, ce qui a pour effet de déplacer le point d'équilibre en faisant monter le taux de change: $k_{i}$ augmente et $k_{j}$ diminue.

\section{Exemple}

Considérons le cas où la fonction d'utilité de chaque compagnie est quadratique

$$
u_{j}(x)=x-a_{j} x^{2} . \quad \forall j
$$


Borch a montré que les contrats Pareto-optimaux sont des traités en quote-part de taux

$$
q_{j}=\frac{\mathrm{I} / k_{j} a_{j}}{\sum_{i=1}^{m} \mathrm{I} / k_{i} a_{i}},
$$

où les $q_{j}$ doivent satisfaire aux inégalités

$$
\begin{aligned}
& q_{j} \supseteq 0 \quad \forall j \\
& \sum_{i=1}^{m} q_{j}=1 \\
& q_{j}^{2} \leqslant \frac{\left(\frac{I}{\partial a_{j}}-R_{j}\right)^{2}+V_{j}}{\left.\sum_{i=1}^{m}\left(\frac{I}{2 a_{i}}-R_{i}\right)\right\}_{j}^{2}+\sum_{i=1}^{m} V_{i}},
\end{aligned}
$$

en désignant par $P_{j}$ la prime pure, par $V_{j}$ la variance de la distribution des risques de $C_{j}$ et par $R_{j}$ la réserve $S_{f}-P_{j}$.

Considérons trois compagnies $C_{1}, C_{2}, C_{3}$, dont les paramètres valent

$$
\begin{array}{lll}
R_{1}=\mathrm{I} & R_{2}=4 & R_{3}=4 \\
P_{1}=\mathrm{I} & P_{2}=2 & P_{3}=2 \\
V_{1}=55 & V_{2}=20 & V_{3}=20 \\
a_{1}=0,0 \mathrm{I} & a_{2}=0,05 & a_{3}=0,05 .
\end{array}
$$

Les utilités initiales valent

$$
U_{j}\left(x_{j}\right)=\frac{I}{4 a_{j}}-a_{j}\left[\left(\frac{I}{2 a_{j}}-R_{j}\right)^{2}+V_{j}\right] .
$$

Donc

$$
\begin{aligned}
& U_{1}\left(x_{1}\right)=0,44 \\
& U_{2}\left(x_{2}\right)=2,2 \\
& U_{3}\left(x_{3}\right)=2,2 .
\end{aligned}
$$

Ces utilités correspondent à des quote-parts extrémales pour $C_{1}$ :

$$
\begin{aligned}
& q_{1}^{\max }=0,802251 \\
& q_{1}^{\text {min }}=0,7577 \text { I } .
\end{aligned}
$$


Posons pour simplifier

$$
\begin{aligned}
& Y_{j}=\left(\frac{I}{2 a_{j}}-R_{j}\right)^{2}+V_{j} ; \\
& Y=\int_{i=1}^{m}\left(\frac{\mathrm{I}}{2 a_{i}}-R_{i}\right)^{2}+\sum_{i=1}^{m} V_{i} .
\end{aligned}
$$

L'utilité après réassurance vaut

$$
U_{j}(\bar{y})=\frac{\mathrm{I}}{4 a_{j}}-a_{j} q_{j}^{2} y . \quad \forall j
$$

En éliminant les paramètres $q_{1}, q_{2}, q_{3}$, nous obtenons l'équation de la surface. Pareto-optimale

$$
\sum_{j=1}^{m}\left(\frac{\frac{I}{4 a_{j}}-U_{j}}{a_{j} Y}\right)^{\frac{1}{2}}-I=0,
$$

ce qui devient dans notre cas

$$
\sqrt{25-U_{1}}+\sqrt{I-U_{2} / 5}+\sqrt{I-U_{3} / 5}=\sqrt{38,16}
$$

La valeur au sens de Shapley transférable (c'est-à-dire sans introduire de poids $k_{j}$ pour le moment) s'obtient en résolvant le système de 2 équations

$$
\begin{gathered}
U_{1}+U_{2}+U_{3}=v(\{\mathrm{I}, 2,3\}) \\
\sqrt{25-U_{1}}+\sqrt{I-U_{2} / 5}+\sqrt{I-U_{3} / 5}=\sqrt{38, I 6}
\end{gathered}
$$

en les quatre inconnues $U_{1}, U_{2}, U_{3}$ et $\bar{v}\{(I, 2,3)\}$, en exprimant que le plan (6) est tangent à la surface (7). Ces calculs donnent

$$
\begin{aligned}
& U_{1}=\phi_{1}(\bar{v})=I, 583 \mathrm{I} 75 \\
& U_{2}=\phi_{2}(\bar{v})=3,07984 \mathrm{I} \\
& U_{3}=\phi_{3}(\bar{v})=3,07984 \mathrm{I} \\
& \bar{v}(\{\mathrm{I}, 2,3\})=7,743957 .
\end{aligned}
$$

En remplaçant dans (5), on obtient

$$
\begin{aligned}
& q_{1}=0,783357 \\
& q_{2}=0,100318 \\
& q_{3}=0,100318 .
\end{aligned}
$$


valeurs non-admissibles car leur somme est inférieure à $\mathrm{I}$. Ce point se trouve au-dessus de la surface Pareto-optimale.

Multiplions donc les paiements de $C_{1}$ par $k_{1}$, ceux de $C_{2}$ par $k_{2}$ et ceux de $C_{3}$ par $k_{3}$. Notons $\phi^{k}(\bar{v})$ et $\bar{v} k(\{I, 2,3\})$ les transformés de $\phi(\bar{v})$ et $\bar{v}(\{I, 2,3\})$ par cette opération. Nous savons que nous pouvons imposer une relation arbitraire aux $k_{f}$. Nous supposons donc que leur somme est égale à $m$.

Ceci nous donne trois équations

$$
\begin{aligned}
\sqrt{25-\phi_{1}^{k}(\bar{v}) / k_{1}}+\sqrt{\mathrm{I}-\phi_{2}^{k}(\bar{v}) / k_{2}} & =\sqrt{\mathrm{I}-\phi_{3}^{k}(v) / k_{3}}=\sqrt{38, \mathrm{I} 6} \\
\phi_{1}^{k}(\bar{v})+\phi_{2}^{k}(\bar{v})+\phi_{3}^{k}(\bar{v}) & =\bar{v}^{k}(\{\mathrm{I}, 2,3\}) \\
k_{1}+k_{2}+k_{3} & =3
\end{aligned}
$$

pour 7 inconnues $\phi_{1}^{k}(\bar{v}), \phi_{2}^{k}(\bar{v}), \phi_{3}^{k}(\bar{v}), k_{1}, k_{2}, k_{3}, \bar{v}^{k}(\{\mathrm{I}, 2,3\})$.

En exprimant que

- la surface Pareto-optimale doit être tangente à l'hyperplan de transférabilité,

- la valeur doit se trouver sur la surface,

et en éliminant les inconnues, nous obtenons après de longs calculs une équation du tè degré en $k_{1}$. Ce polynôme possède trois racines négatives et une seule racine positive

$$
\begin{aligned}
& k_{1}=0,7763 \mathrm{I} 3 \\
& k_{2}=\mathrm{I}, \mathrm{III} 68_{+} \\
& k_{3}=\mathrm{I}, \mathrm{III} 68+ \\
& \phi_{1}^{k}(\bar{v})=\mathrm{I}, \mathrm{I} 69078 \\
& \phi_{2}^{k}(\bar{v})=3,099 \mathrm{I} 99 \\
& \phi_{3}^{k}(\bar{v})=3,099 \mathrm{I} 99 \\
& \bar{v}^{k}(\{\mathrm{I}, 2,3\})=7,367+77 .
\end{aligned}
$$

En divisant ces valeurs par $k_{1}, k_{2}, k_{3}$ et en remplaçant dans (5), il vient

$$
\begin{array}{ll}
q_{1}=0,784648 & U_{1}(\bar{v})=1,505937 \\
q_{2}=0,107676 & U_{2}(\bar{v})=2,787842 \\
q_{3}=0,107676 & U_{3}(\bar{v})=2,787842
\end{array}
$$


Comme on pouvait s'y attendre, $C_{1}$, ayant le moins peur du risque, va prendre à sa charge une quote-part importante des sinistres. En contrepartie, elle va évidemment exiger une compensation monétaire. On peut montrer que celle-ci doit être égale à

$$
y_{j}(0)=q_{j} \sum_{i=1}^{m}\left(\frac{\mathrm{I}}{2 a_{i}}-S_{i}\right)-\left(\frac{\mathrm{I}}{2 a_{j}}-S_{j}\right) .
$$

Il vient

$$
y_{2}(0)=y_{3}(0)=2,029856
$$

Donc $C_{1}$ va percevoir au total

$$
-y_{1}(0)=y_{2}(0)+y_{3}(0)=4,0597 \text { I } 2 \text {. }
$$

\section{\$5. Un Nouveau Concept de Valeur}

Le $\S$ précédent nous a permis d'isoler un traité de la surface Pareto-optimale. Un certain nombre de critiques peuvent cependant être formulées à l'égard du concept de valeur de Shapley (voir [7]). Le défaut le plus grave du modèle est que l'axiome 3 de linéarité n'est certainement pas vérifié car les compagnies évaluent leur situation au moyen de fonctions d'utilité, par définition non-additives: l'utilité résultant de la signature de deux traités n'est pas égale à la somme des utilités partielles. C'est pourquoi nous avons défini (dans [6]) un nouveau concept de solution, basé directement sur les jeux à utilités non-transférables, en généralisant un modèle de marchandage de Nash ([8]).

Les axiomes permettant d'isoler un traité sont les suivants.

I) La solution n'est pas affectée par une transformation linéaire effectuée sur les utilités.

2) La solution est fonction de tous les sous-traités relatifs aux coalitions d'effectifs inférieurs à $m$; chaque sous-traité satisfait auc relations $(I)$ et $(2)$.

3) Tout jeu symétrique a une solution symétrique.

4) La solution ne change pas si nous retirons de l'espace du jeu tout point autre que le paiement initial et la solution elle-même. 
Pour simplifier les notations, posons

$$
\begin{gathered}
K=\left\{k_{1}, \ldots, k_{m} \mid \text { les } k_{j} \text { sont liées par une relation }\right\} \\
\left.y_{j}(S)=y_{j}\left\{x_{i} \mid i \varepsilon S\right\}\right) \quad \cdot j \varepsilon S \\
U_{j}(S)=U_{j}\left[y_{j}(S)\right]
\end{gathered}
$$

$U_{j}(S)$ est l'utilité pour $C_{j}$ d'un traité signé par les membres d'une coalition $S$.

Supposons qu'à un moment quelconque de la négociation un premier groupe $S_{1}$ de joueurs soit arrivé à un traité optimal $\bar{y}\left(S_{1}\right)$, permettant aux joueurs $C_{i}\left(i \varepsilon S_{1}\right)$ d'obtenir une utilité $U_{i}\left(S_{1}\right)$ tandis qu'un autre groupe $S_{2}$ (tel que $S_{1} \cap S_{2}=\phi$ ) a conclu un traité optimal $\bar{y}\left(S_{2}\right)$ donnant à $C_{l}\left(l \varepsilon S_{2}\right)$ une utilité $U_{l}\left(S_{2}\right)$. Ces deux groupes se réunissent en vue de signer un traité global $\bar{y}^{x}\left(S_{1} \cup S_{2}\right)$ (le symbole $U$ a ici un sens légèrement différent d'une réunion; $S_{1} U S_{2}$ veut dire ", $S_{1}$ se joint à $S_{2}{ }^{\prime \prime}$. Le - est placé pour rappeler que le résultat ne dépend pas uniquement de l'ensemble $S_{1} U S_{2}$ mais aussi de la manière dont cette coalition s'est formée, c'est-àdire de $S_{1}$ et $S_{2}$ ). Si les deux groupes ne parviennent pas à se mettre d'accord sur un traité $\bar{y}\left(S_{1} U S_{2}\right)$, ils retombent nécessairement au point de départ de la négociation

$$
\begin{array}{ll}
U_{i}\left(S_{1}\right) & \forall C_{i} \varepsilon S_{1} \\
U_{l}\left(S_{2}\right) & \forall C_{l} \varepsilon S_{2} .
\end{array}
$$

Pour cette raison, ce paiement est appelé le point de désaccord.

Lemme: Le traité $\bar{y}^{x}\left(S_{1} U S_{2}\right)$ est l'unique point tel que

$$
\psi^{x}=\max \psi=\max _{K} \prod_{i \mathbf{s} S_{1}}\left[U_{i}\left(S_{1} U S_{2}\right)-U_{i}\left(S_{1}\right)\right] \prod_{l \mathbf{s} S_{2}}\left[U_{l}\left(S_{1} U S_{2}\right)-U_{l}\left(S_{2}\right)\right]
$$

THÉoRÈME 7: Il existe un et un seul traité $\bar{y}(M)$ satisfaisant aux 4 axiomes. Il peut s'obtenir par la récurrence

$$
\begin{aligned}
& y_{f}(\{j\})=x_{j}
\end{aligned}
$$

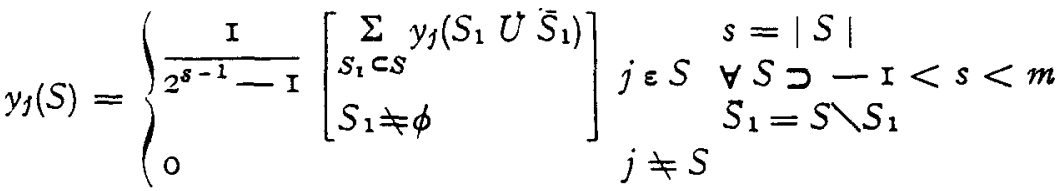




$$
y_{j}(M)=\frac{I}{2^{s-1}-I}\left[\begin{array}{l}
\sum_{S_{1}{ }_{1}} y_{j}\left(S_{1} \cup \bar{S}_{1}\right) \\
S_{1} \neq \phi
\end{array}\right] \begin{aligned}
& m=|M| \\
& \bar{S}_{1}=M / S_{1} \\
& j=\mathrm{I}, \ldots, m
\end{aligned}
$$

où, à chaque étape, $y_{f}\left(S_{1} \dot{U} \overline{S_{1}}\right)$ est obtenu par la solution de (8) dont le point de désaccord est

$$
\begin{cases}U_{i}\left(S_{1}\right) & V i \approx S_{1} \\ U_{l}\left(\vec{S}_{1}\right) & \forall l \approx S_{1}\end{cases}
$$

La solution se construit par induction sur le nombre de joueurs d'une coalition: il faut successivement calculer la valeur de tous les ensembles comprenant 2 compagnies, 3 compagnies, etc..., pour arriver finalement à la coalition $M$. Supposons que nous ayons calculé les valeurs pour toutes les coalitions dont l'effectif ne dépasse pas $s$ - I et construisons le traité optimal pour un ensemble $S$ de $s$ partenaires. $S$ contient $2^{s-1}$ - I sous-coalitions (strictes) $S_{1}$ pour lesquelles il existe un sous-traité. Pour chaque $S_{1}$, nous calculons par (8) un traité

$$
\bar{y}\left[S_{1} \cup\left(S \backslash S_{1}\right)\right] \text {. }
$$

L'utilité accordée à une compagnie ne diminue pas par cette opération: il est en effet facile de montrer que (8) fournit toujours un $U_{l}\left(S_{1} U S_{2}\right)$ supérieur ou égal à $U_{i}\left(S_{1}\right)$. Au plus le point de désaccord est élevé pour un joueur, au plus la solution de (8) lui est favorable. Contrairement au modèle de Shapley, le bénéfice de la coalition est ici réparti entre les compagnies suivants leurs forces respectives.

Nous obtenons ainsi $2^{s-1}-\mathrm{I}$ traités, en général différents, que nous résumons par une moyenne arithmétique. Nous avons de la sorte déterminé un traité optimal unique pour $S$. La solution du jeu s'obtient pour $S=M$.

Ce concept de valeur tient donc compte de l'ordre de formation du marché: chaque joueur s'allie avec d'autres compagnies ou ensembles de compagnies, de telle sorte qu'après un nombre fini de jonctions, $M$ soit formée et un traité partiel soit conclu. Toutes les possibilités de groupement sont envisagées et interviennent avec la même force dans le traité final. La solution est l'espérance mathé- 
matique des traités partiels lorsque toutes les formations de coalition sont équiprobables.

\section{Exemple}

Reprenons l'exemple du $\S 4$.

$$
\text { Utilités initiales } \quad \begin{aligned}
& U_{1}(\{I\})=0,44 \\
& U_{2}(\{2\})=2,2 \\
& U_{3}(\{3\})=2,2 .
\end{aligned}
$$

Ensembles de deux compagnies.

Coalition $\{I, 2\}$. La maximisation du produit

$$
\begin{aligned}
& {\left[U_{1}(\{I, 2\})-U_{1}(\{I\})\right]\left[U_{2}(\{I, 2\})-U_{2}(\{2\})\right] } \\
= & {\left[-a_{1} q_{1}^{2} Y+a_{1} Y_{1}\right]\left[-a_{2} q_{2}^{2} Y+a_{2} Y_{2}\right] }
\end{aligned}
$$

conduit, après élimination de $q_{2}$, à une équation du troisième degré en $q_{1}$ :

$$
q_{1}^{3}-\frac{3}{2} q_{1}^{2}+\frac{Y-Y_{1}-Y_{2}}{2 Y} q_{1}+\frac{Y_{1}}{2 Y}=0 .
$$

La résolution de cette équation donne

$$
\begin{array}{ll}
q_{1}=0,877593 & U_{1}(\{\mathrm{I}, 2\})=\mathrm{I}, \mathrm{I} 24759 \\
q_{2}=0,122407 & U_{2}(\{\mathrm{I}, 2\})=2,677553 .
\end{array}
$$

Coalition $\{I, 3\}$. En vertu de la symétrie entre $C_{2}$ et $C_{3}$, il vient

$$
\begin{array}{ll}
q_{1}=0,877593 & U_{1}(\{\mathrm{I}, 3\})=1, \mathrm{I} 24759 \\
q_{3}=0,122407 & U_{3}(\{\mathrm{I}, 3\})=2,677553 .
\end{array}
$$

Coalition $\{2,3\}$

$$
\begin{array}{ll}
q_{2}=0,5 & U_{2}(\{2,3\})=2,7 \\
q_{3}=0,5 & \left.\left.U_{3}(\} 2,3\right\}\right)=2,7 .
\end{array}
$$

Coalition $\{I, 2,3\}$. Le système formé par les équations (I) et (2) s'écrit après résolution par la méthode des multiplicateurs de Lagrange,

$$
\begin{aligned}
& q_{1}\left(q_{2}^{2} Y-Y_{2}\right)=q_{2}\left(q_{1}^{2} Y-Y_{1}\right) \\
& q_{1}\left(q_{3}^{2} Y-Y_{3}\right)=q_{3}\left(q_{1}^{2} Y-Y_{1}\right) \\
& q_{1}+q_{2}+q_{3}=I
\end{aligned}
$$

et peut se résoudre par approximations successives. 


$$
\begin{aligned}
& \{\mathrm{I}, 2\} U\{3\} q_{1}=0,78335^{8} \\
& q_{2}=0,102926 \\
& q_{3}=0,1137 \text { I } 6 \\
& \{1,3\} U\{2\} q_{1}=0,78335^{8} \\
& q_{2}=0, \operatorname{II} 37 \mathrm{I} 6 \\
& q_{3}=0,102926 \\
& \{2,3\} U\{I\} q_{1}=0,794826 \\
& q_{2}=0,102587 \\
& q_{3}=0,102587 \text {. }
\end{aligned}
$$

$$
\text { Solution optimale } \begin{array}{|l|}
\hline q_{1}=0,787 \mathrm{I} 8 \\
q_{2}=0, \mathrm{I06} 4 \mathrm{I} \\
q_{3}=0, \mathrm{I06} 4 \mathrm{I} \\
U_{1}(\{\mathrm{I}, 2,3\})=\mathrm{I}, 354042 \\
U_{2}(\{I, 2,3\})=2,839563 \\
U_{3}(\{I, 2,3\})=2,839563 \\
\hline y_{3}(0)=-3,91792 \\
y_{2}(0)=I, 95896 \\
y_{3}(0)=I, 95896 .
\end{array}
$$

La solution est donc légèrement moins favorable à la première compagnie.

\section{BIBLIOGRAPHIE}

[1] BORCH, K., "The safety loading of reinsurance premiums" Skandinavsk Aktuarietidskrift, 43 (1960), pp. 163-184.

[2] BoRch, K., "Reciprocal reinsurance treaties" ASTIN Bulletin, I (1960) Pp. $170-19 \mathrm{r}$.

[3] Borch, K., "Equilibrium in a reinsurance market" Econometrica, 30 (I96̈2), pp. 424-444.

[4] BoRCH, K., "Reciprocal Reinsurance Treaties seen as a Two-Person Cooperative Game" Skandinavsk Aktuarietidskrifi, 43 (1960), pp. 29-58.

[5] DU MOUCHEL, W., "The Pareto-optimality of an n-company reinsurance treaty" Skandinavsk Aktuarietidskrift, 5 I (I968), pp. I65-I70.

[6] LEMAIRE, J., "A new concept of value for games without transferable utilities" The International Journal of Game Theory 1974.

[7] LemaIRe, J., "Sur la valeur au sens de Shapley" Cahiers au C.E.R.O. vol. $14, \mathrm{n}^{\circ} \mathrm{I}, 1974$.

[8] Nash, J., "The Bargaining Problem" Econometrica, I8 (1950), pp. 155-162. 
[9] Shapley, L. S., "A value for n-person games" Contributions to the theory" of games, Vol. II. (Annals of Mathematics Studies, 28) pp. 307-3I7.

[Io] Shapley, L. S., "Utility comparison and the theory of games" La décision. Comptes rendus d'un colloque du C.N.R.S. à Aix-en-Provence. 1967, pp. $25 \mathrm{I}-263$ 